\title{
Automedicação em gestantes de alto risco: foco em atenção farmacêutica
}

\section{Automedication in high risk pregnant: focus on pharmaceutical care}

\author{
Sandna Larissa Freitas dos Santos ${ }^{1}$. Hérick Hebert da Silva Alves ${ }^{2}$. Cinara Vidal Pessoa ${ }^{3}$. Maria Luíza Bezerra \\ de Macedo Arraes ${ }^{4}$. Karla Bruna Nogueira Torres Barros ${ }^{5}$. \\ 1 Farmacêutica, Centro Universitário Católica de Quixadá, Ceará, Brasil. 2 Discente do curso de Farmácia, Centro Universitário \\ Católica de Quixadá, Ceará, Brasil. 3 Farmacêutica, Mestre em Saúde da criança e do Adolescente pela Universidade Estadual do \\ Ceará (UECE), Fortaleza, Ceará, Brasil. 4 Farmacêutica, Doutoranda em Desenvolvimento de Medicamentos pela Universidade \\ Federal do Ceará (UFC), Fortaleza, Ceará, Brasil. 5 Farmacêutica, Mestre em Ensino na saúde pela Universidade Estadual do \\ Ceará (UECE), Fortaleza, Ceará, Brasil.
}

\section{RESUMO}

Introdução: O mercado farmacêutico está em crescentes mudanças, elevando a prática da automedicação em gestantes, necessitando de intervenções e investigações dos efeitos decorrentes do uso de medicamentos. Objetivo: verificar o índice da automedicação em gestantes de alto risco atendidas na Policlínica Francisco Carlos Cavalcante Roque- Quixadá-CE. Métodos: Estudo observacional, transversal, consistindo em uma abordagem predominantemente quantitativa realizado em março a maio de 2016, com 80 gestantes. Foi utilizado o teste Exato de Fisher e o teste do Qui-quadrado, com inserção no software Microsoft Excel para viabilizar o processamento e análise das respostas obtidas. Resultados: A automedicação foi relatada por 33,7\% das gestantes, e 11,4\% delas afirmaram sentir-se mal no uso de Dipirona, Ibuprofeno e Dimenidrinato. Os medicamentos mais frequentes utilizados pelas pacientes, na forma de automedicação, foram: 27\% Ibuprofeno, $18 \%$ Dipirona, 6\% Bromoprida, $40 \%$ Paracetamol, 9\% Dimenidrinato. Os principais fatores motivadores do uso foram cefaleia (40,7\%), êmese (20,3\%) e náuseas (39\%). A automedicação por decisão pessoal foi a mais prevalente $(94,9 \%)$, porém, $6,1 \%$ declaram utilizar medicamento por indicação de terceiro (mãe). Conclusão: $\mathrm{O}$ alto índice da automedicação pelas gestantes refere a necessidade de intervenções educativas direcionadas ao uso seguro de medicamentos.

Palavras-chave: Gravidez de alto risco. Automedicação. Uso de medicamentos.

\section{ABSTRACT}

Introduction: The pharmaceutical market is under increasing changes, increasing the practice of self-medication in pregnant women, necessitating interventions and investigations of the effects arising from the use of drugs. Objective: To verify the index of self-medication in high-risk pregnant women attended at the Francisco Carlos Cavalcante Roque-Quixadá-CE Polyclinic. Methods: A cross-sectional, observational study consisting of a predominantly quantitative approach conducted in March to May 2016, with 80 pregnant women. Fisher's exact test and the chi-square test were used, with insertion in Microsoft Excel software to enable the processing and analysis of the answers obtained. Results: Self-medication was reported by $33.7 \%$ of pregnant women, and $11.4 \%$ of them reported feeling sick with Dipirone, Ibuprofen and Dimenhydrin. The most frequent drugs used by the patients in the form of self-medication were: $27 \%$ Ibuprofen, $18 \%$ Dipyrone, $6 \%$ Bromopride, $40 \%$ Paracetamol, $9 \%$ Dimenhydrin, the main motivating factors were headache $(40.7 \%)$, emesis $(20.3 \%)$ and nausea $(39 \%)$. Self-medication by personal decision was the most prevalent (94.9\%), but $6.1 \%$ reported using medication by indication of third (mother). Conclusion: The high index of self-medication by pregnant women refers to the need for educational interventions aimed at the safe use of medications.

Keywords: High risk pregnancy. Self-medication. Drug utilization.

Autor correspondente: Sandna Larissa Freitas dos Santos, Rua Major Pedro Sampaio, 1166, Rodolfo Teófilo, Fortaleza, Ceará. CEP: $60430-185$. Telefone: +55 85 99766-4341. E-mail: sandy.lary@hotmail.com

Conflito de interesses: Não há qualquer conflito de interesses por parte de qualquer um dos autores.

Recebido em: 08 Set 2017; Revisado em: 21 Nov 2017; Aceito em: 09 Dez 2017. 


\section{INTRODUÇÃO}

O uso de medicamentos na gestação representa, ainda hoje, um desafio para a medicina, visto que grande parte dos fármacos atravessa a barreira placentária e a maioria não foi testada clinicamente em gestantes, podendo gerar riscos ao feto. Ao mesmo tempo, a automedicação, o fenômeno da medicalização e a falta de informação sobre os riscos do mau uso de medicamentos são problemas adicionais. ${ }^{1}$

A gestação oferece, no entanto, barreiras éticas e técnicas à realização de ensaios clínicos, neste sentido, a farmacovigilância tem aportado conhecimento, por meio da investigação e avaliação dos efeitos decorrentes do uso agudo e crônico de medicamentos. Isto tem permitido não somente a determinação do impacto dos defeitos congênitos na população mundial, mas também a elaboração de estratégias para reduzir a incidência de malformações congênitas, possivelmente causadas por medicamentos. ${ }^{2,3}$

Pelos riscos potenciais ao feto em desenvolvimento, uma vez que a maioria dos fármacos administrados tem a capacidade de atravessar a placenta e expor o feto em desenvolvimento a seus efeitos farmacológicos e/ou teratogênicos, o uso dessas substancias na gestação merece essa especial atenção, devendo ser, por princípio, evitada. Os efeitos sobre o feto dependem do fármaco ou substância utilizadas, das condições clínicas da paciente, da época de exposição durante a gestação, da frequência e da dose total, redundando potencialmente em aspectos teratogênicos ou com consequências farmacológicas e toxicológicas diversas. ${ }^{4,5}$

$\mathrm{O}$ forte crescimento da indústria farmacêutica fez com que os medicamentos ocupassem um lugar de destaque para a cura das doenças e alívio de sintomas. Contudo, o uso sem limites de medicamentos acarreta consequências negativas para a mãe e para o feto, aumento de efeitos colaterais e reações adversas, que muitas vezes são gravíssimas. ${ }^{1}$

Nesse contexto, o estudo tem como objetivo averiguar a utilização de medicamentos por automedicação pelas gestantes atendidas em um serviço de referência ao pré-natal de alto risco no interior do estado do Ceará, verificando qual o perfil das doenças apresentadas pelas pacientes, identificando os medicamentos e suas respectivas classes farmacológicas mais usadas, além de classificar conforme FDA (Food and Drug Administration) em categorias de risco para o uso de medicamentos na gravidez em um serviço de referência do interior do Ceará.

\section{MATERIAL E MÉTODOS}

O estudo foi do tipo observacional, transversal, consistindo em uma abordagem predominantemente quantitativa. Foi desenvolvido na Policlínica Francisco Carlos Cavalcante Roque, Quixadá-CE no período de março a maio de 2016. O serviço realizou o atendimento de 95 gestantes cadastradas pelo Sistema Único de Saúde (SUS) e encaminhadas da atenção básica. Os critérios de inclusão foram gestantes que estavam à espera da consulta de pré-natal no momento da pesquisa, com idade superior a 18 anos, aptas e conscientes para responder as informações contidas no questionário, em conformidade com a participação na pesquisa e as prescrições geradas a partir das consultas.

Das 95 gestantes, 10 eram menores de idade e 5 recusaram a participação, com isso foram entrevistadas 80 gestantes de alto risco, onde cada uma assinou o Termo de consentimento livre e esclarecido, garantindo os aspectos legais na participação da pesquisa. Foi realizado uma entrevista, utilizando como instrumento um questionário (Figura 1) em que foi traçado o perfil sócio econômico e colhidas informações relacionadas à doença, automedicação, motivos do uso dos medicamentos e possíveis ocorrências de reações adversas.

Assim, após estruturação dos dados, as informações pertinentes foram analisadas estatisticamente e os dados foram inseridos no banco de dados do software Microsoft Excel para viabilizar o processamento e análise das respostas obtidas. A abordagem quantitativa foi avaliada pelo programa de SPSS e pelo Teste Exato de Fisher. ${ }^{6}$

Os resultados foram descritos de forma quantitativa como frequência absoluta e percentual dos entrevistados em relação às suas variáveis sociodemográficas, econômicas, e de forma absoluta e percentual, em relação às variáveis farmacoterapêuticas. Nestes casos, o teste Exato de Fisher e o teste do Qui-quadrado foram utilizados para avaliar se estas variáveis influenciaram significativamente na prevalência de distribuição das entrevistadas. Foi adotado, como nível de significância, valores de $\mathrm{p}$ inferiores a 0,05 .

Foram respeitados os requisitos quanto à confidencialidade $\mathrm{e}$ sigilo das informações, de acordo com as determinações feitas pela Resolução $466 / 12^{7}$ e as usuárias não foram submetidas a qualquer tipo de experimentação. O estudo foi avaliado pelo Comitê de Ética em Pesquisa do Centro Universitário Católica de Quixadá e foi aprovado de acordo com o protocolo $\mathrm{N}^{\mathrm{o}}$. 1.506.719.

\section{RESULTADOS}

As características socioeconômicas das pacientes estão descritas na Tabela 1. Quanto à idade gestacional, apresentaram uma faixa de 8 a 39 semanas com 75,4\% em 21 semanas. Das 80 gestantes entrevistadas, o uso de cigarro foi afirmado por $5(6,2 \%)$ e nenhuma relatou o uso de drogas ilícitas.

A utilização de medicamentos durante a gravidez pela prática da automedicação foi relatada por 27 (33,75\%) gestantes, e 3 $(11,1 \%)$ delas afirmaram sentir-se mal ao tomarem os seguintes medicamentos: Dipirona, Ibuprofeno e Dimenidrinato.

A automedicação foi relatada por $27(33,75 \%)$ gestantes, distribuídos em: Paracetamol (40,0\%), Ibuprofeno (27\%), Dipirona $(18,0 \%)$, Dimenidrinato $(9,0 \%)$ e Bromoprida (6,0\%) (Gráfico 1). 
Figura 1. Questionário usado na entrevista com as gestantes atendidas na Policlínica Francisco Carlos Cavalcante Roque, Quixadá-Ceará, Nordeste, Brasil-2016.

\section{QUESTIONÁRIO}

Pesquisa: "Automedicação em gestantes de alto risco atendidas em um Serviço de Referência do interior do Ceará" Questionário No:

Data:

1 - Identificação:

Idade:

Idade Gestacional:

2 - Estado civil:

semanas ( ) não sabe informar

\begin{tabular}{|c|c|c|}
\hline ) Solteiro & ( & ) União formal \\
\hline ) Viúvo/a & ( & ) Separado/a ou Divorciado/a \\
\hline ) Casado (a) & ( & Não sabe informar \\
\hline
\end{tabular}

Anos de estudo: ( ) Não alfabetizada ( ) Não sabe informar

4 - Situação Habitacional:

Tipo de Moradia: ( ) Própria ( ) Alugada ( ) Cedida ( ) Familiares ( ) Outros

( ) Não sabe informar

5 - Renda mensal:

$\mathrm{R} \$$

( ) Não sabe informar

6 - Em caso de doenças recorre a:

( ) Médico/Enfermeiro ( ) Vizinho ( ) Farmácia ( ) Plantas medicinais ( ) Não sabe informar

7 - Faz uso de cigarro?

( ) Sim ( ) Não ( ) Não sabe informar

8 - Faz uso de drogas?

( ) Sim ( ) Não ( ) Não sabe informar

9 - Durante a gestação já fez ou faz algum uso de medicamento, sem a prescrição médica?

( ) Sim ( ) Não ( ) Não sabe informar

10 - Qual a patologia de alto risco que apresenta?

( ) Hipertensão Gestacional

( ) Pré-eclâmpsia/eclampsia

( ) Anemia

( ) Diabetes

( ) Hemorragias

( ) Lúpus Eritematoso Sistêmico

( ) Sifilis

( ) Herpes simples vírus (HSV)

( ) Não sabe informar

11 - Fonte de orientação para utilização de medicamentos:

( ) Vizinhos ( ) Família ( ) Farmacêutico ( ) Enfermeiro ( ) Outros

( ) Não sabe informar

12 - $\mathrm{O}$ uso de algum medicamento durante a gravidez já causou algum efeito indesejado ou mal-estar:

( ) Sim ( ) Não ( ) Não sabe informar

Qual(is)?

13 - Já recebeu alguma cartilha ou informativo sobre o uso de medicamentos em gestantes:

( ) $\operatorname{Sim~(~)~Não~(~)~Não~sabe~informar~}$

Se sim, onde:

14 - Medicamentos usados pela automedicação durante a gestação:

\begin{tabular}{|l|l|l|l|l|}
\hline Nome & $\begin{array}{l}\text { Forma } \\
\text { farmacêutica }\end{array}$ & $\begin{array}{l}\text { Classe } \\
\text { farmacêutica }\end{array}$ & Para que usa? & Quem indicou? \\
\hline & & & & \\
\hline & & & & \\
\hline
\end{tabular}

15 - Tem conhecimentos dos riscos ocorridos pelo uso de medicamentos na automedicação?

( ) Sim ( ) Não ( ) Não sabe informar 
Tabela 1. Características socioeconômicas das gestantes atendidas na Policlínica Francisco Carlos Cavalcante Roque, Quixadá- Ceará, Nordeste, Brasil.

\begin{tabular}{|c|c|c|}
\hline \multicolumn{3}{|l|}{ Características sócio econômicas } \\
\hline Idade & Frequência & $\%$ \\
\hline 18 a 35 anos & 17 & $21,2 \%$ \\
\hline Acima de 35 anos & 63 & $70,75 \%$ \\
\hline \multicolumn{3}{|l|}{ Estado civil } \\
\hline Solteira & 5 & $6,25 \%$ \\
\hline Casada & 54 & $67,5 \%$ \\
\hline União Formal & 21 & $26,2 \%$ \\
\hline \multicolumn{3}{|l|}{ Escolaridade } \\
\hline Não alfabetizada & 3 & $3,7 \%$ \\
\hline Ensino fundamental completo & 19 & $23,7 \%$ \\
\hline Ensino médio completo & 45 & $56,3 \%$ \\
\hline Ensino superior completo & 13 & $16,3 \%$ \\
\hline \multicolumn{3}{|l|}{ Renda Familiar } \\
\hline Até 1 salário & 24 & $30,0 \%$ \\
\hline 2 salários & 28 & $35,0 \%$ \\
\hline 3 salários & 6 & $7,5 \%$ \\
\hline 4 salários & 1 & $1,2 \%$ \\
\hline Não sabe informar & 21 & $26,3 \%$ \\
\hline \multicolumn{3}{|l|}{ Situação Habitacional } \\
\hline Alugada & 24 & $30,0 \%$ \\
\hline Própria & 56 & $70,0 \%$ \\
\hline \multicolumn{3}{|l|}{ Uso de Cigarro } \\
\hline Sim & 5 & $6.2 \%$ \\
\hline Não & 75 & $93,8 \%$ \\
\hline \multicolumn{3}{|l|}{ Uso de drogas ilícitas } \\
\hline Sim & 0 & $0,0 \%$ \\
\hline Não & 80 & $100,0 \%$ \\
\hline \multicolumn{3}{|l|}{ Uso de Bebidas alcoólicas } \\
\hline Sim & 12 & $16,0 \%$ \\
\hline Não & 68 & $84,0 \%$ \\
\hline
\end{tabular}

Gráfico 1. Medicamentos utilizados por automedicação pelas gestantes atendidas na Policlínica Francisco Carlos Cavalcante Roque, Quixadá- Ceará, Nordeste, Brasil-2016.

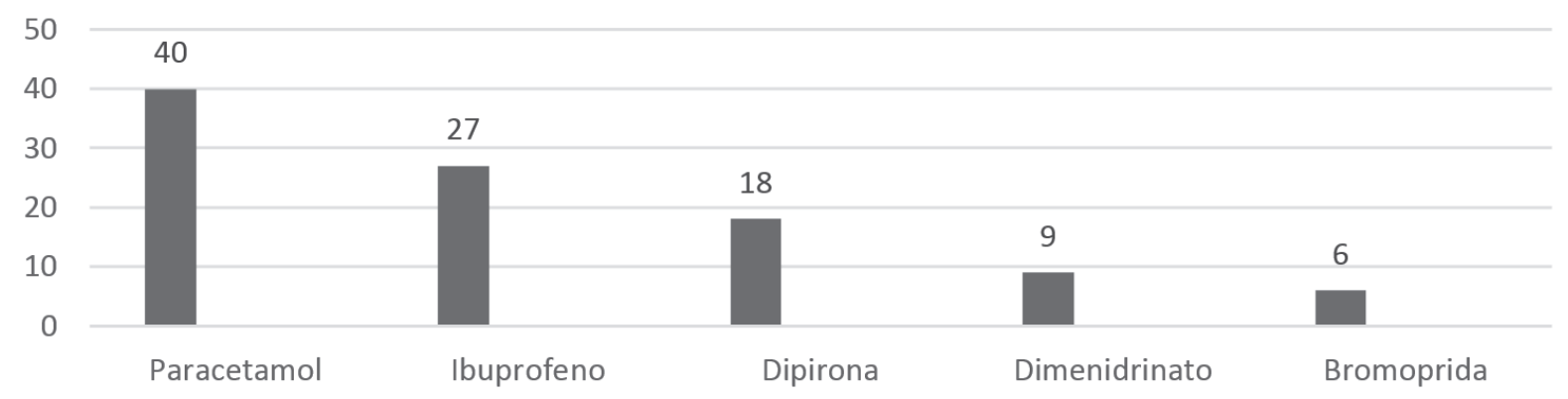


As participantes relataram que as queixas de uso desses medicamentos foram: cefaleia $(40,7 \%)$, êmese $(20,3 \%)$ e náuseas (39\%) e segundo $6,1 \%$ das gestantes a indicação partiu da mãe e as demais por decisão pessoal 94,9\%. De acordo com a classificação terapêutica dos medicamentos estão os AINES $(85,0 \%)$ e Anti-eméticos (15,0\%).

Foi observado que $39(48,75 \%)$ gestantes afirmaram ter como fonte de orientação sobre o uso de medicamentos a consulta com o profissional médico, $28(35 \%)$ na farmácia pelo farmacêutico, 12 (15\%) no posto de saúde com o enfermeiro, e $1(1,25 \%)$ afirmou buscar a internet. As doenças de alto risco das gestantes estão descritas no Gráfico 2.
A utilização de plantas medicinais foi afirmada por $56(70,0 \%)$ gestantes, dentre elas, 14 (25,0\%) utilizaram "erva-cidreira" (Lippia alba), 10 (17,5\%) "erva-doce" (Pimpinella anisum), 9 (16\%) "capim-santo" (Cymbopogon citratus), 8 (14,5\%) "boldo" (Peumus boldus) seguidos por 8 (14,5\%) "camomila" (Matricaria recutita/Chamomilla recutita), e 7 (12\%) "hortelãda-folha-miúda (Mentha spp.) (Gráfico 3).

As mulheres entrevistadas foram também questionadas em relação à educação em saúde, em que $80,0 \%$ das gestantes afirmaram ter recebido orientações durante o pré-natal somente pelos profissionais enfermeiros, e ainda que nenhuma recebeu algum material informativo sobre o uso racional de medicamentos a gestantes de alto risco.

Gráfico 2. Doenças relatadas pelas gestantes atendidas na Policlínica Francisco Carlos Cavalcante Roque, Quixadá- Ceará, Nordeste, Brasil- 2016.

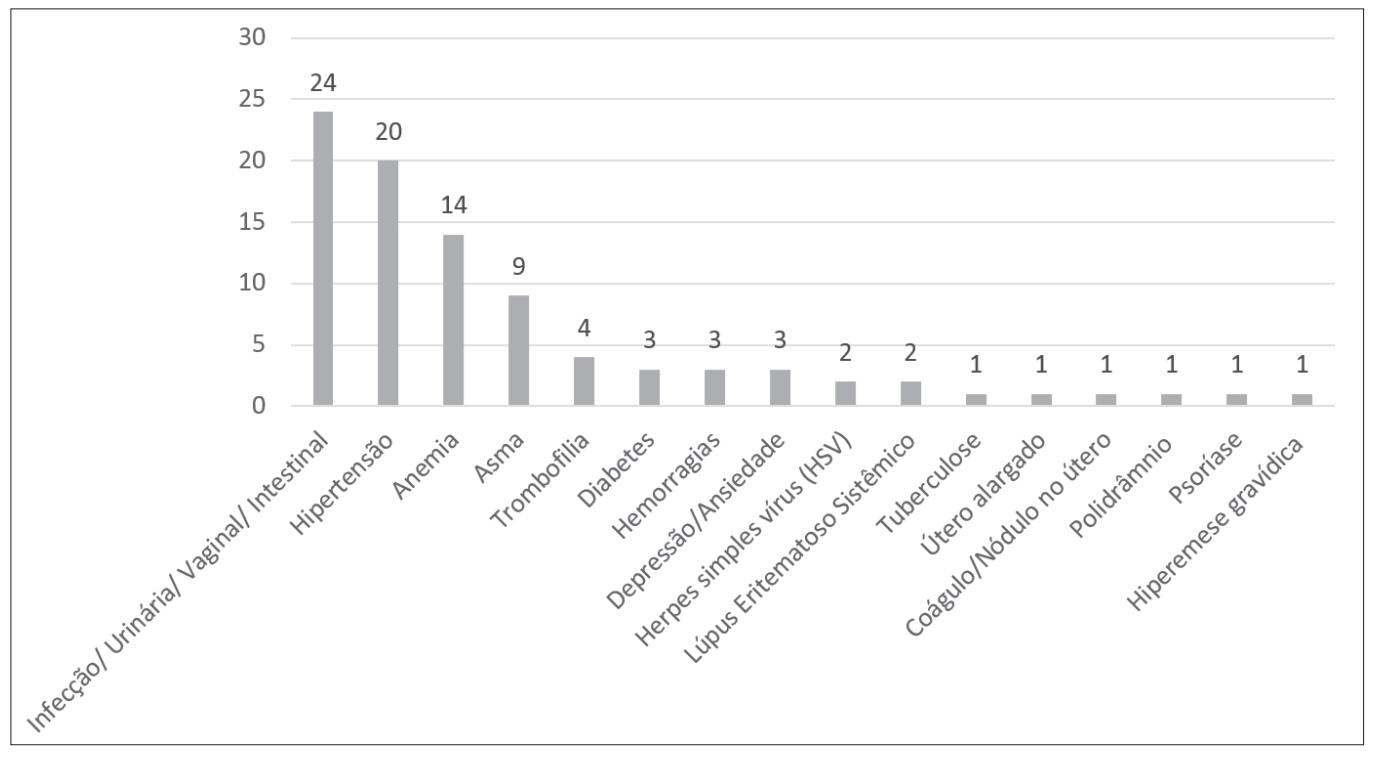

Gráfico 3. Uso de plantas medicinais relatadas pelas gestantes atendidas na Policlínica Francisco Carlos Cavalcante Roque, Quixadá- Ceará, Nordeste, Brasil- 2016.

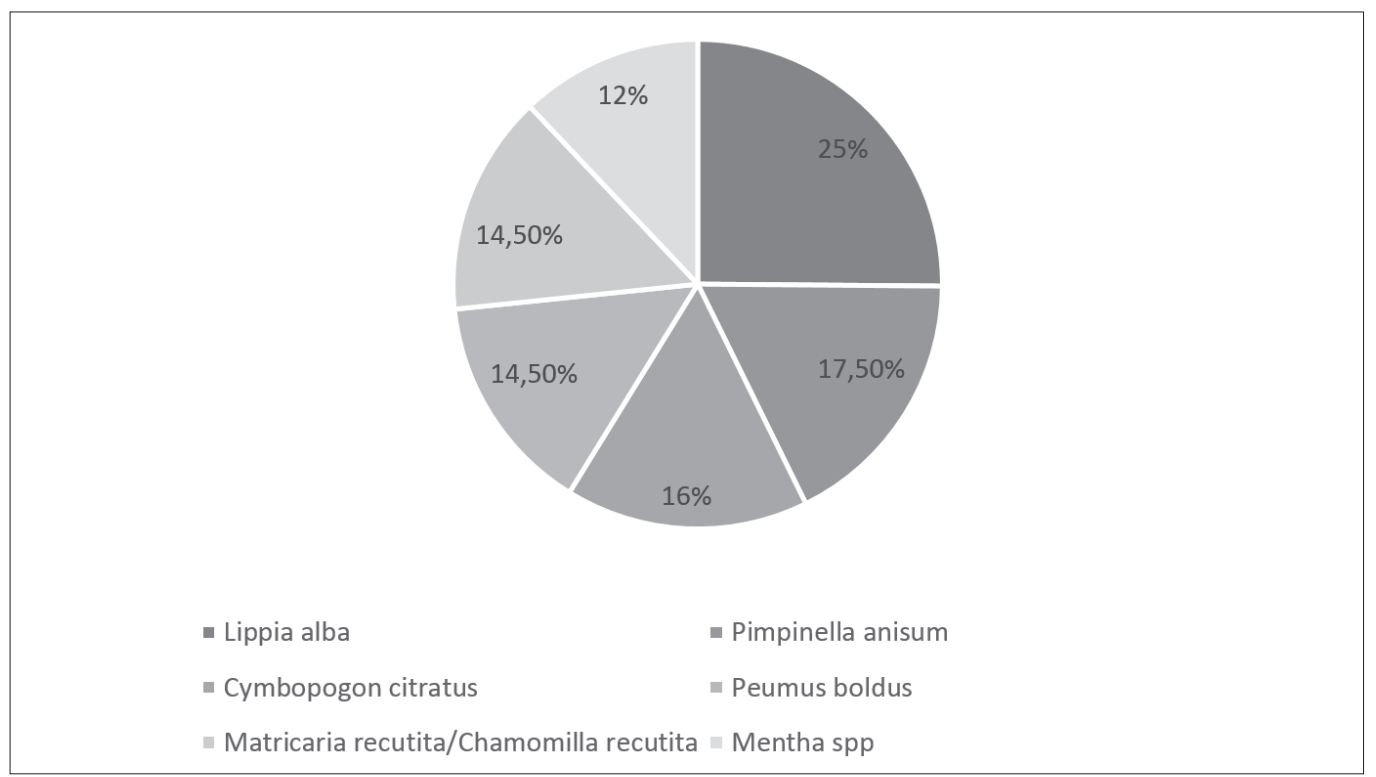




\section{DISCUSSÃO}

Pôde-se observar que houve maior prevalência de gestantes entre a faixa etária de 29 a 39 anos, divergindo dos dados preconizados pelo Ministério da Saúde sendo a idade inferior a 15 anos ou acima de 35 anos um dos fatores geradores de risco na gestação. ${ }^{5}$

A maior prevalência de mulheres casadas (60,0\%) se relaciona com a residência no interior do estado, que buscam companheiros de forma mais evidente. A escolaridade de ensino médio completo $(56,2 \%)$ se correlata a maior predominância de mulheres com renda de 2 salários $(35,0 \%)$ e morando em casa própria $(70,0 \%)$.

Assemelhando aos dados da pesquisa de Beserra et al. ${ }^{8}$ que entrevistou 145 gestantes com faixa etária variando de 13 a 42 anos, a faixa etária predominante se concentrou entre 28 e 32 anos, com $54(37,24 \%)$ mulheres. Quanto à escolaridade, 53 $(36,55 \%)$ gestantes apresentaram o ensino médio completo. A avaliação da distribuição da renda mensal mostrou que $64(44,14 \%)$ recebiam até 1 salário mínimo e 70 (48,28\%) entre 1 e 2 salários mínimos. Os autores ainda relataram que a baixa escolaridade entre gestantes repercute na qualidade e na realização da assistência pré-natal, divergindo dos dados encontrados no presente estudo.

Melo et al. ${ }^{9}$ apresentou um estudo em 205 gestantes com média de idade de 23,7 anos, 80 (40\%) estavam na faixa etária entre 20 e 25 anos, $70(34,1 \%)$ possuíam o Ensino Fundamental Incompleto e $95(46,4 \%)$ eram casadas. Quando comparado o grau de instrução e uso de medicamento durante a gestação não houve diferença estatisticamente significativa. ${ }^{9}$

O terceiro trimestre de gravidez foi o período mais relatado pelas gestantes, equivalendo da $27^{\circ}$ a $40^{\circ}$ semana (43\%). Dados semelhantes aos encontrados no estudo de Melo et al., ${ }^{10}$ no qual as mulheres que estavam no terceiro trimestre foram as que mais relataram ter utilizado ou estar usando algum medicamento, e os mais consumidos foram o sulfato ferroso $(92-45,0 \%)$ e o paracetamol $(89-43,4 \%)$.

Acredita-se que o número encontrado nesta pesquisa a respeito da automedicação $(33,75 \%)$ seja elevado. Visto que a saúde pública não tem recebido recursos orçamentários adequados e cuja estrutura organizacional representada pelo SUS não alcançou patamar suficientemente eficiente, pode-se esperar que as pessoas das classes que dependem desse sistema estariam mais sujeitas a se automedicar. Comparativamente, num estudo realizado no Estado do Paraná foi encontrado um valor baixo $(8,2 \%)$, levando em consideração as 245 gestantes entrevistadas. ${ }^{9}$ Esses dados evidenciam que a prática do uso de medicamentos sem orientação profissional é uma realidade presente independente do estado, escolaridade e cultura, se estendendo por diversas parte do país. No estudo de Nunes et al. $^{10}$ foi visto que o dimenidrinato e Ibuprofeno foram causadores de efeitos eméticos nas gestantes avaliadas.

A classificação em categorias de risco para o uso de medicamentos na gravidez de acordo com a FDA é definida de acordo com o risco de um medicamento causar defeitos congênitos e outros efeitos na gestação. Dos medicamentos usados 0 foi encontrado na Categoria $\mathrm{A}$ - é bastante remota a possibilidade de dano fetal; 2 (40\%) na Categoria B - prescrição com cautela; 2 (40\%) em Categoria $\mathrm{C}$ - prescrição com risco; $1(10 \%)$ em Categoria D - prescrição com Alto Risco e 0 em Categoria $\mathrm{X}$ - prescrição com Perigo (contraindicada).

Rocha et al. ${ }^{11}$ mostrou que o tabagismo esteve presente em $11,3 \%$ das gestações, sendo $75,7 \%$ até o final da gestação, em um total de 336 gestantes. A ingestão de bebidas alcoólicas durante a gravidez foi afirmada por $16 \%$ das mulheres, com $59,6 \%$ delas permanecendo no consumo até o final do terceiro trimestre. No mesmo estudo, não foi possível uma associação com significado estatístico entre o consumo de álcool e fumo durante a gestação e a presença de malformações fetais.

O uso de cigarro, relatado por $6,2 \%$ das gestantes no estudo, reflete negativamente na formação do feto, principalmente nos três primeiros meses de gestação e eleva a incidência de problemas na saúde da mãe, tornando o organismo mais vulnerável a comorbidades cardíacas e infecções, correspondendo aos achados das patologias de alto risco.

Ainda, no estudo de Rocha et al. ${ }^{11}$ foi visto que o consumo de pelo menos um medicamento na gestação teve uma prevalência de $96,6 \%$ e uma média de 2,8 medicamentos por gestante, em um total de 336 gestantes. A automedicação foi referida por 37 (11,3\%) gestantes, evidenciando um dado inferior ao encontrado no presente estudo o qual foi de 27 $(33,7 \%)$.

Da-se importância aos tratamentos maternos com AINEs $(14,1 \%)$, que têm sido associados, com frequência, à vasoconstrição do ducto arterioso fetal, hipertensão arterial pulmonar e inibição da agregação plaquetária. Estudos sobre os efeitos dos anti-inflamatórios demonstram alta incidência de síndrome de hipertensão pulmonar persistente no recém-nascido e de anormalidades na hemostasia. ${ }^{12}$

A maioria dos AINEs é classificada como (C), mas não devem ser utilizados no terceiro trimestre por causarem constrição do ducto arterioso fetal que pode acarretar em hipertensão arterial pulmonar. Com menor frequência, perdas fetais, baixo peso ao nascer e alterações glomerulares e de coagulação foram relatadas. Mesmo assim, alguns pacientes necessitam de tratamento com anti-inflamatório, casos em que damos preferência a prednisona em baixas doses. Indometacina é amplamente utilizada na prevenção do parto prematuro, mas tem sido relacionada a casos de enterocolite. $\mathrm{O}$ uso de ibuprofeno foi relacionado a casos de defeitos glomerulares acarretando insuficiência renal. ${ }^{13} \mathrm{Na}$ contextualização do risco e benefício dessa utilização, ver-se então que apesar desses efeitos, diante da patologia da mãe a prescrição é evidenciada.

$\mathrm{Na}$ literatura foi encontrado que o paracetamol é o analgésico de primeira escolha na gravidez e que a dipirona possui restrições específicas, portanto sua utilização deveria ser evitada durante a gestação. ${ }^{12}$ 
Foi visto que o terceiro trimestre de gravidez foi o período mais relatado pelas gestantes, equivalente a $27^{\circ}$ a $40^{\circ}$ semana $(43 \%)$.

É possível verificar que 39\% apresentaram como doenças de alto risco a infecção distribuídas em urinária, vaginal e intestinal, e ainda 25\% apresentaram Hipertensão Gestacional. Carvalho e Araújo ${ }^{13}$ apresentam um estudo com 612 puérperas no pós-parto imediato, e que de acordo com o prontuário médico, $368(60,2 \%)$ das entrevistadas apresentaram algum tipo de morbidade na gravidez por ocasião do internamento, sendo as mais frequentes: a doença hipertensiva específica da gravidez $135(36,7 \%)$ e a pré-eclâmpsia 107 (29,1\%).

De acordo com o Ministério da Saúde, as complicações hipertensivas na gravidez são a maior causa de morbidade e mortalidade materna e fetal, e ocorrem em cerca de $10 \%$ de todas as gestações. ${ }^{5}$

De acordo com a revisão de literatura realizada, a planta mais utilizada pelas gestantes é a camomila, que de acordo com as referências bibliográficas, tem uso contraindicado durante a lactação e gestação, por possuir ação abortiva e ser um relaxante uterino. Em segundo lugar, aparece a hortelã, que também apresenta efeito abortivo, e em seguida, a melissa que não deve ser administrada na gravidez e lactação. Em quarto lugar, aparece o boldo, que pode acarretar redução do peso fetal e apresenta ação abortiva, contraindicado no primeiro trimestre de gestação. ${ }^{14-15}$

Dados semelhantes foram encontrados em um estudo desenvolvido no âmbito da Estratégia Saúde da Família (ESF) de Cuité-PB, no período de abril a outubro de 2010, com 64 gestantes atendidas nas unidades, verificou-se que 16 (25\%) fizeram uso de algum tipo de planta medicinal. Todas as nove plantas medicinais citadas pelas gestantes foram consideradas contraindicadas, sendo o boldo $(62,5 \%)$, erva-cidreira $(18,75 \%)$ e canela $(12,5 \%)$ as mais utilizadas na forma de chás. Constipação, dor, febre e ansiedade foram algumas das motivações para o uso destas plantas medicinais. ${ }^{16}$

Beserra et al. ${ }^{9}$ entrevistaram 145 gestantes que se apresentavam para consulta pré-natal no serviço de Obstetrícia da Policlínica Dr. Luiz Santos Filho e em dez Unidades Básicas de Saúde do município de Gurupi-TO. As classes de medicamentos mais usadas foram: antianêmicos, analgésicos, antiespasmódicos e antibióticos. Entre as gestantes, 50 (34,5\%) relataram a utilização de plantas medicinais, sendo a erva-cidreira a

\section{REFERÊNCIAS}

1. Araujo DD, Leal MM, Santos EJ, Leal LB. Consumption of medicines in high-risk pregnancy: evaluation of determinants related to the use of prescription drugs and self-medication. Braz J Pharm. 2013;49(3):491-9.

2. Rodrigues AV, Terrengui LC. Uso de medicamentos durante a gravidez. Rev Enferm UNISA. 2006;7(1):9-14.

3. Guerra GC, Silva AQ, França B, Assunção PM, Cabral RX, Ferreira AA. Utilização de medicamentos durante a gravidez na mais citada, por 41 (28\%) gestantes, seguida da camomila 29 (20\%), capim-santo $21(14 \%)$ e erva-doce $3(6 \%)$ na forma de fitoterápico. Dos medicamentos isentos de prescrição médica destacaram-se o paracetamol $\left(\right.$ Tylenol $\left.^{\circledR}\right)$ e a dipirona (Novalgina $\left.^{\circledR}\right) .105(72,41 \%)$ gestantes disseram não concordar com a automedicação, enquanto 40 (27,59\%) gestantes concordaram com a prática.

A prática de educação em saúde direcionada ao enfermeiro o evidencia como o profissional mais próximo dessa população nos serviços de saúde. Observa ainda que a fonte de orientação sobre o uso de medicamentos se dá pela comunicação com o profissional médico no momento da consulta, com o Farmacêutico durante a dispensação dos medicamentos na farmácia, com o enfermeiro nas unidades básicas de saúde e na internet como meio de fácil obtenção das informações sobre as substâncias, mesmo sem a real credibilidade.

Cabe ressaltar que esta pesquisa foi realizada através da coleta de informações envolvendo a lembrança das mulheres, o que pode levar a subjetividade dos dados, pois a recordação de fatos passados, de certa forma, pode não corresponder totalmente com a realidade. Além disso, o tempo limitado das entrevistas realizadas após a consulta das gestantes pela pressa em sair do estabelecimento, se apresenta como restrição na pesquisa.

\section{CONCLUSÕES}

A automedicação foi reportada pelas entrevistadas de forma elevada, sendo mais presente a classe dos anti-inflamatórios não esteroidais, o que gera preocupação à saúde materna e fetal pois eleva os riscos de efeitos colaterais e interações medicamentosas, podendo causar efeitos teratogênicos e tóxicos ao feto e ainda foi observado que a utilização de plantas medicinais requer atenção e orientação de seu uso adequado. Diante dos resultados encontrados neste estudo, pode-se concluir que a medicalização na gravidez é uma realidade, já que há um elevado consumo de medicamentos durante a gestação sem orientação profissional do uso adequado e dos riscos teratogênicos implicados.

Nesse contexto, os resultados desse estudo poderão subsidiar o planejamento de intervenções educativas dirigidas a gestantes, contribuindo para o uso seguro e racional de medicamentos na gestação, com atuação do profissional farmacêutico visando a incidência de condições de alto risco.

cidade de Natal, Rio Grande do Norte, Brasil. Rev Bras Ginecol Obstet. 2008;30(1):12-18.

4. Santos DT, Campos CS, Duarte ML. Perfil das patologias prevalentes na gestação de alto risco em uma maternidade escola de Maceió, Alagoas, Brasil. Rev Bras Med Fam Comunidade. 2014;9(30):13-22.

5. Brasil. Ministério da Saúde. Secretaria de Atenção à Saúde. Departamento de Ações Programáticas Estratégicas. Gestação de 
alto risco: manual técnico. 5. ed. Brasília: Ministério da Saúde; 2010.

6. Brites R. Manual de técnicas e métodos quantitativos. Tomo I. Lisboa: Instituto Nacional de Administração; 2007.

7. Brasil. Conselho Nacional de Saúde. Resolução nº66 de 12 de dezembro de 2012 [Internet]. Brasília: CNS; 2012 [acesso em: 23 mai 2017]. Disponível em: http://bvsms.saude.gov.br/bvs/saudelegis/ cns/2013/res0466_12_12_2012.html

8. Beserra FP, Paiva SG, Sousa SF, Lopes SP, Azevedo DA, Borges JC. Perfil de utilização de medicamentos em gestantes assistidas em serviço público de saúde de Gurupi, Tocantins. Revista Cereus. 2014;6(1):71-91.

9. Melo SC, Pelloso SM, Carvalho MD, Oliveira NL. Uso de medicamentos por gestantes usuárias do Sistema Único de Saúde. Acta Paul Enferm. 2009;22(1):66-70.

10. Nunes AM, Bayer VM, Felisbino FE, Castro AA, Gazola AC, Martins LP. A utilização de medicamentos por gestantes internadas em um Hospital da região sul catarinense: caracterização e avaliação dos riscos envolvidos. Rev Ciênc Cidadania. 2015;1(1):57-68.
11. Rocha RS, Bezerra SC, Lima JW, Costa FS. Consumo de medicamentos, álcool e fumo na gestação e avaliação dos riscos teratogênicos. Rev Gaucha Enferm. 2013;34(2):37-45.

12. Fonseca CS, Viloria MI, Repetti L. Alterações fetais induzidas pelo uso de antiinflamatórios durante a gestação. Cienc Rural. 2002;32(4):529-34.

13. Menezes MS, Medeiros MM, Barbosa PB, Ferreira AA, Medeiros CA. Uso de medicamentos por gestantes atendidas no Hospital da Polícia Militar - Mossoró/RN. Rev Bras Farm. 2014;95(1):512-29.

14. Carvalho VC, Araújo TV. Adequação da assistência pré-natal em gestantes atendidas em dois hospitais de referência para gravidez de alto risco do Sistema Único de Saúde, na cidade de Recife, Estado de Pernambuco. Rev Bras Saúde Matern Infant. 2007;7(3): 309-17.

15. Melo A, Anhesi N, Rosa LG, Pereira AC. Uso de plantas medicinais na gestação. [Internet]. RETEC. 2016;9(2):101-9.

16. Pontes SM, Souza AP, Barreto BF, Oliveira HC, Oliveira LB, Saraiva AM, et al. Utilização de plantas medicinais potencialmente nocivas durante a gestação na cidade de Cuité-PB. Com Ciências Saúde. 2012;23(4):305-11.

\section{Como citar:}

Santos SL, Alves HH, Pessoa CV, Arraes ML, Barros KB. Automedicação em gestantes de alto risco: foco em atenção farmacêutica. Rev Med UFC. 2018 jul-set;58(3):36-43. 